\title{
Theoretical Evaluation of Bifurcation in Dependence on Resonance Frequency in Two-Coil Inductive Power Transfer
}

\author{
Košík M. ${ }^{1}$, Fajtl R. ${ }^{2}$, Lettl J. ${ }^{3}$ \\ Department of Electric Traction and Drives, Faculty of Electrical Engineering \\ Czech Technical University in Prague \\ Prague, Czech Republic \\ ${ }^{1}$ kosikmi1@fel.cvut.cz; ${ }^{2}$ fajtlrad@fel.cvut.cz; ${ }^{3}$ lettl@,fel.cvut.cz
}

\begin{abstract}
Operation of two-coil inductive power transfer (IPT) system is affected by bifurcation phenomenon. In this paper, bifurcation in dependency on resonance frequency is examined. At first, voltage equations are analyzed, and impedance and transfer function derived. In next step, condition for resonance frequency is stated, which is followed by bifurcation analysis. Resulting bifurcation map is analytically described. Lastly, impacts of bifurcation on resonance frequency choice are evaluated using impedance and transfer function.
\end{abstract}

Keywords - Contactless power transfer; inductive power transfer; transfer function; bifurcation.

\section{INTRODUCTION}

Term contactless power transfer (CPT) refers to a power transfer between two objects without any contact which could transfer the power. Multiple variants of CPT exists, however, they all have a similar general principle: Power is transmitted from the transmitter by some oscillating field. Provided that the field, which is generated by the transmitter, has the same frequency as the resonance frequency of the receiver is, the power transfer from transmitter to receiver is established. Types of CPT can be distinguished by a medium [1], [2]:

- Acoustic energy transfer (AET). Power is transferred by mechanical oscillations, which usually has a form of sound. In [3] can be found an evaluation of the current state of AET.

- Electromagnetic power transfer (EMPT). Power is transferred by an electromagnetic field. This method can be used for power transfer over the longest distances - in [4] is discussed the use of MPT for space application.

- Capacitively coupled power transfer (CCPT). Power is transferred through an electric field. Operational distances are relatively short due to the small coupling capacitance. In [3] can be found a description of CCPT principle and comparison with inductively coupled power transfer.

- Inductive power transfer (IPT) - Power is transferred through a magnetic field between two coils. IPT is currently the most widely used method to achieve contactless power transfer. Overview and modern trends can be found in [5], [6].
This paper focuses on two-coil IPT. This setup was for the first time used by N. Tesla [7], at the end of 19th century and elaborated by University of Auckland's group of scientists led by A. W. Green, J. T. Boys and G. Covic in the 1990s [5]. For theoretical description, they used circuit theory [8]. Two coils IPT is used for short range applications (usually up to 50 $\mathrm{cm}$ ). This setup is currently most widely used in various applications, with power ranging from $10 \mathrm{~W}$ (home electronics) to $100 \mathrm{~kW}$ (industrial applications, electric vehicle charging) [6]. Operating frequency is dependent on the application and bridged distance, with values in the range $10 \mathrm{kHz}$ to $100 \mathrm{kHz}$ and beyond [6].

For comparison, four-coil setup developed by a team led by Marin Soljačić at Massachusetts Institute of Technology (MIT) in 2007, allows transfer over

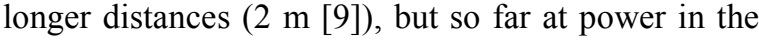
range of hundreds of Watts and frequencies in around $8 \mathrm{MHz}[10]$.

Each two-coil IPT system has two operation regions, which differ in the number of the zero phase angle (ZPA) frequencies - frequencies at which the phase angle between the input voltage and current is equal to zero. In the first (bifurcation-free) region there is only one ZPA frequency, equal to the resonance frequency. When IPT system moves into the second (bifurcation) region, this one ZPA frequency splits into three. ZPA frequency division is called bifurcation. System control and operation is substantially different for the bifurcation-free region or the bifurcation region. Therefore bifurcation phenomenon must be considered at IPT design.

In [8], the dependency of the bifurcation phenomena on IPT system quality factors is described. However, bifurcation is also significantly affected by choice of the resonance frequency, which is examined in this paper. The analysis consists of the following steps: At first, the two-coil IPT setup and its voltage equations are described. Main instruments of analysis - impedance as seen by source and the voltage transfer function is derived from voltage equations. In the third step, the condition for resonance is listed. This condition leads to bifurcation analysis, which is also a part of the third step. Lastly, results are evaluated in conclusion, and future work is outlined. 


\section{TWO-COIL IPT SETUP AND OPERATION}

In Fig. 1 the two-coil IPT is depicted. Main elements are transfer coils (or windings), represented by inductances $L_{1}, L_{2}$ and resistances $R_{1}, R_{2}$. They are coupled by mutual inductance $M\left(L_{1}=L_{1 \sigma}+M\right.$, $L_{2}=L_{2 \sigma}+M ; L_{1 \sigma}, L_{2 \sigma}$ are leakage inductances). To achieve resonance, both sides are compensated by capacitors $\left(C_{1}, C_{2}\right)$, which are chosen in such way that primary and secondary side has same resonance frequency. Capacitors can be placed in parallel or in series, each option has its advantages and disadvantages [5]. In this case, series-series (SS) compensation is chosen. Circuit is powered by sinusoidal input voltage $v_{i}$ and loaded by pure resistance $R_{L}$. Output voltage $v_{o}$ is related to the load resistance $\left(v_{o}=R_{L} i_{2}\right)$.

Figure 1: Two-coil IPT schematics.

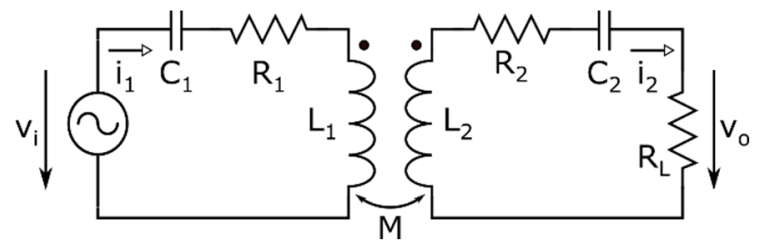

System operation can be illustrated by the following example: Imagine two strings. If each of them is tuned to a different tone, the second one stands still when the first one is played. However, when you tune both of them to the same tone, the second one starts vibrating when you play the first one. IPT coils correspond to the strings and tuning is achieved by capacitive compensation. Values of the SS compensation capacitors can be calculated from (1), (2), where $\omega_{0}$ is common resonance angular frequency. Equations (1), (2) can be derived from condition for resonance operation (13).

$$
\begin{aligned}
& C_{1}=\frac{1}{\omega_{0}^{2} L_{1}} \\
& C_{2}=\frac{1}{\omega_{0}^{2} L_{2}}
\end{aligned}
$$

Primary and secondary windings can be described by voltage equations (3), (4).

$v_{i}(t)=R_{1} i_{1}(t)+L_{1} \frac{d i_{1}(t)}{d t}+\frac{1}{C_{1}} \int_{0}^{t} i_{1}(\tau) d \tau-M \frac{d i_{2}(t)}{d t}$

$M \frac{d i_{1}(t)}{d t}=\left(R_{2}+R_{L}\right) i_{2}(t)+L_{2} \frac{d i_{2}(t)}{d t}+\frac{1}{C_{2}} \int_{0}^{t} i_{2}(\tau) d \tau$

Assuming powering from ideal sinusoidal source, voltage equations for primary and secondary side can be expressed by phasors:

$$
\begin{gathered}
\widehat{V}_{\iota}=R_{1} \widehat{I_{1}}+j \omega L_{1} \widehat{I_{1}} \frac{1}{j \omega C_{1}} \widehat{I_{1}}-j \omega M \widehat{I_{2}} \\
j \omega M \widehat{I_{1}}=R_{2} \widehat{I_{2}}+j \omega L_{2} \widehat{I_{2}}+\frac{1}{j \omega C_{2}} \widehat{I_{2}}+R_{L} \widehat{I_{2}}
\end{gathered}
$$

\section{CIRCUIT ANALYSIS}

In this section, the two-coil IPT setup is analyzed in terms of impedance and voltage transfer function.

\section{A. Impedance}

Circuit impedance $\hat{Z}$ as seen from the source can be expressed from equations (5), (6) as:

$$
\hat{Z}=\frac{\left(R_{1}+j\left(\omega L_{1}-\frac{1}{\omega C_{1}}\right)\right) \cdot\left(R_{2}+R_{L}+j\left(\omega L_{2}-\frac{1}{\omega C_{2}}\right)\right)+\omega^{2} M^{2}}{R_{2}+R_{L}+j\left(\omega L_{2}-\frac{1}{\omega C_{2}}\right)}
$$

For easier comprehension, substitutions in TABLE I are performed. Impedance equation (7) then changes into:

$$
\hat{Z}=\frac{\left(R_{p}+j X_{p}\right) \cdot\left(R_{S}+j X_{S}\right)+X_{M}^{2}}{R_{S}+j X_{S}}
$$

TABLE I: SUBSTITUTIONS

\begin{tabular}{|c|c|l|}
\hline & Resistance & Reactance \\
\hline $\begin{array}{c}\text { Primary } \\
\text { side }\end{array}$ & $R_{p}=R_{1}$ & $j X_{p}=j \omega L_{1}+j \frac{1}{\omega C_{1}}$ \\
\hline $\begin{array}{c}\text { Secondary } \\
\text { side }\end{array}$ & $R_{S}=R_{2}+R_{L}$ & $j X_{S}=j \omega L_{2}+\frac{1}{j \omega C_{2}}$ \\
\hline $\begin{array}{c}\text { Mutual } \\
\text { inductance }\end{array}$ & - & $j X_{M}=j \omega M$ \\
\hline
\end{tabular}

The impedance can be divided into real part and imaginary part:

$$
\hat{Z}=R_{p}+\frac{R_{S} X_{M}^{2}}{R_{S}^{2}+X_{S}^{2}}+j\left(X_{p}-\frac{X_{M}^{2} X_{S}}{R_{S}^{2}+X_{S}^{2}}\right)
$$

A two-coil IPT system with parameters tabulated in TABLE II was simulated in Matlab 2015b to verify and illustrate the analysis. Primary side and secondary side always have the same resonance frequency. Values of compensation capacities are calculated from the desired common resonance frequency.

TABLE II: SIMULATED IPT SYSTEM PARAMETERS

\begin{tabular}{|c|l|c|}
\hline Sym. & Parameter & Value \\
\hline$R_{1}$ & Primary resistance & $0.15 \Omega$ \\
\hline$R_{2}$ & Secondary resistance & $0.05 \Omega$ \\
\hline$R_{L}$ & Load resistance & $2.5 \Omega$ \\
\hline$N_{1}$ & N. of primary winding turns & 30 \\
\hline$N_{2}$ & N. of secondary winding turns & 15 \\
\hline$p$ & Turns ratio & 2 \\
\hline$L_{a}$ & Primary leakage inductance & $100 \mu \mathrm{H}$ \\
\hline$L_{b}$ & Secondary leakage inductance & $10 \mu \mathrm{H}$ \\
\hline$M$ & Mutual inductance & $5 \mu \mathrm{H}$ \\
\hline
\end{tabular}

Waveforms of impedance amplitude and phase are different for the bifurcation-free region and bifurcation region. In Fig. 2, the impedance waveforms for resonance frequency $f_{0}=20 \mathrm{kHz}$ are illustrated, which lies in bifurcation-free region. Impedance (Fig. 2 Top) has capacitive character at low operating frequency $f$ (phase shift depicted in Fig. 2 Bottom is equal to $-\frac{\pi}{2}$ ). With increasing $f$ the impedance amplitude drops exponentially, until it reaches resonance region. In this region amplitude drop accelerates until minimal value $0.305 \Omega$ at $f_{0}$ is reached. Phase starts increasing until it reaches zero, 
Figure 2: Impedance in bifurcation-free region.
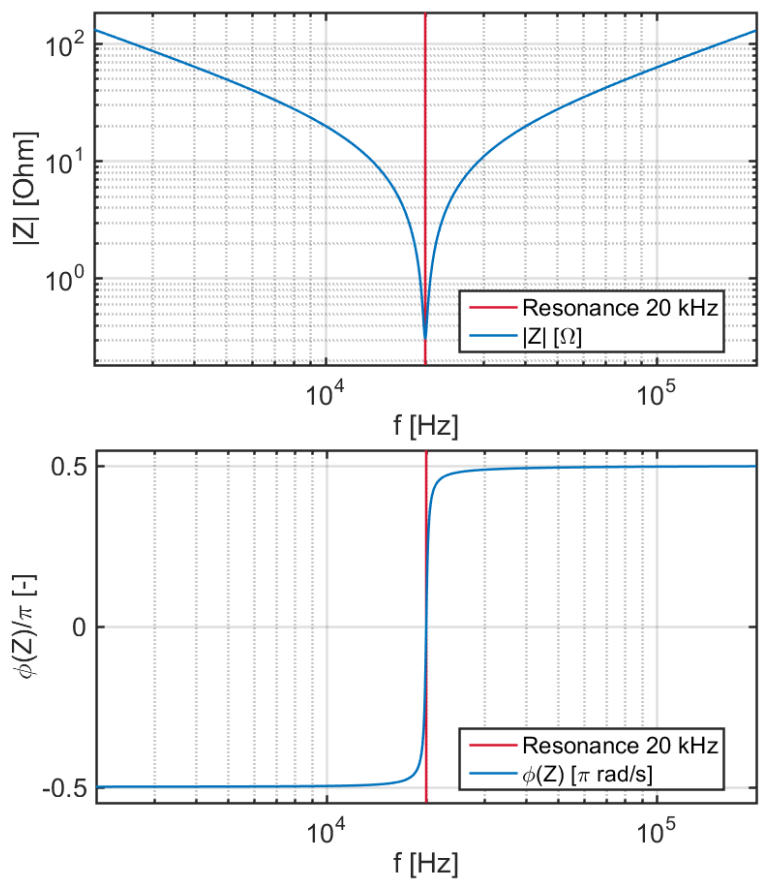

Figure shows dependency of impedance $\boldsymbol{Z}$ on operational frequency $\boldsymbol{f}$ in bifurcation-free region. Top - Impedance amplitude. Bottom Impedance phase.

Figure 3: Impedance in bifurcation region.
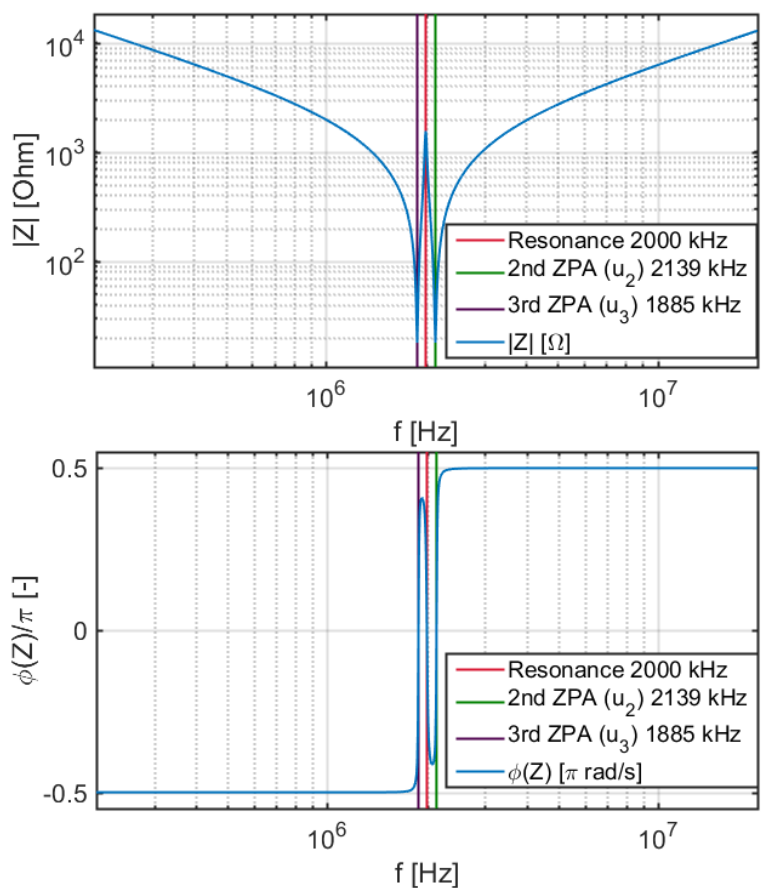

Figure depicts dependency of impedance $\boldsymbol{Z}$ on operational frequency $\boldsymbol{f}$ in bifurcation-free region. Top - Impedance amplitude. Bottom - Impedance phase.

also at $f_{0}$. With further increase of $f$, IPT system gets out of resonance region, amplitude starts growing exponentionally, and phase reaches $\frac{\pi}{2}$, corresponding to inductive character.

Impedance waveforms for bifurcation region are similar to bifurcation region (Fig. 3), when operation frequency $f$ is not close to resonance frequency $f_{0}$. However, in resonance region, additional ZPA frequencies manifest themselves. The lowest amplitude $(18 \Omega)$, is now at $2^{\text {nd }}$ and $3^{\text {rd }}$ ZPA frequency, and at resonance frequency $2 \mathrm{MHz}$ it steeply rises to value $1.55 \mathrm{k} \Omega$. Impedance phase is equal to zero at all ZPA frequencies, however, it switches polarity between each transition through zero (Fig. 3 Bottom).

\section{B. Voltage Transfer Function}

Relation of input voltage $v_{i}$ and output voltage $v_{o}$ can be conveniently described by transfer function. Transfer function is defined by equation (10). It is derived from voltage equations (3), (4).

$$
G(s)=\frac{V_{o}(s)}{V_{i}(s)}
$$

The transfer function can be expressed in the frequency domain (11) or in Laplace domain (12).

$$
\begin{aligned}
& G(j \omega)=\frac{j R_{L} X_{M}}{\left(R_{p}+j X_{p}\right)\left(R_{S}+j X_{s}\right)+X_{M}^{2}} \\
& G(s)=\frac{k_{3} s^{3}}{q_{3} s^{4}+q_{3} s^{3}+q_{2} s^{2}+q_{1} s+1} \\
& k_{3}=R_{L} C_{1} C_{2} M \\
& q_{4}=L_{1} L_{2} C_{1} C_{2}-C_{1} C_{2} M^{2} \\
& q_{3}=R_{1} L_{2} C_{1} C_{2}+R_{S} L_{1} C_{1} C_{2} \\
& q_{2}=R_{1} R_{s} C_{1} C_{2}+L_{1} C_{1}+L_{2} C_{2} \\
& q_{1}=R_{1} C_{1}+R_{s} C_{2}
\end{aligned}
$$

Bode characteristics of the transfer function for the bifurcation-free region are depicted in Fig. 4 and for bifurcation region in Fig. 5. IPT system was operated at same frequencies as in the case of impedance $(20 \mathrm{kHz}$, $2 \mathrm{MHz}$ ). Transfer function magnitude shows high damping outside the resonance region in both cases (Fig. 4, Fig. 5 Top). Magnitude in bifurcation-free region has a single peak $6.11 \mathrm{~dB}$ at the resonance frequency. This means that output voltage is with respect to input voltage amplified 2.2 times. Similarly as in the case of impedance, in bifurcation region, the transfer function magnitude has the highest values ($8.70 \mathrm{~dB}$ in both cases) at $2^{\text {nd }}$ and $3^{\text {rd }} \mathrm{ZPA}$ frequencies. At resonance frequency, magnitude drops to $-28.01 \mathrm{~dB}$.

Transfer function phase is illustrated in Fig. 4 and Fig. 5 Bottom. In bifurcation-free region, output voltage precedes the input voltage by $\frac{3 \pi}{2}$ at frequencies under $f_{0}$. Approaching $f_{0}$, phase shift decreases until it reaches $\frac{\pi}{2}$ at $f_{0}$. The phase decline continues with increasing $f$ until it stabilizes at $-\frac{\pi}{2}$ - output voltage is delayed after input voltage. In bifurcation region, transition between predeceased and delayed phase occurs in steps, which correspond with additional ZPA frequencies. First step is from $\frac{3 \pi}{2}$ to $\frac{\pi}{2}$ and at $3^{\text {rd }} \mathrm{ZPA}$ frequency the phase has value $\pi$. Second step is from $\frac{\pi}{2}$ to $-\frac{\pi}{2}$ at $3^{\text {rd }}$ ZPA frequency the phase has value 0 . Phase shift at resonance frequency remains $\frac{\pi}{2}$, as in the case of bifurcation-free region.

\section{RESONANCE AND BIFURCATION}

As it was described in section II., power transfer between transmitter and receiver windings occurs when resonance frequencies $f_{1}$ (primary), $f_{2}$ (secondary) are 
Figure 4: Voltage transfer function in bifurcation-free region.
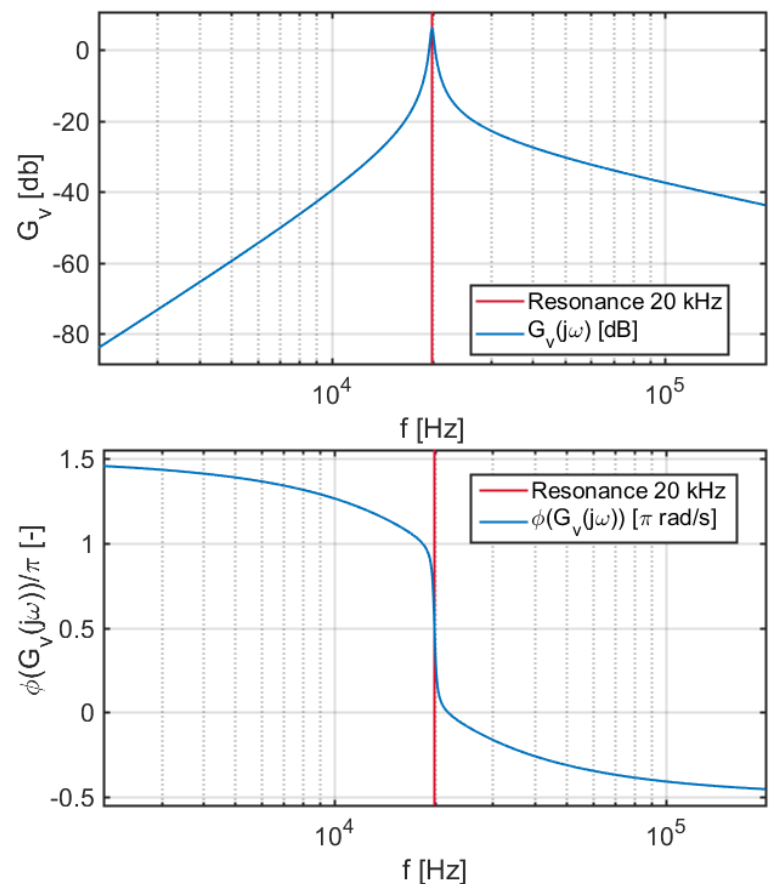

Figure shows dependency of voltage transfer function $\boldsymbol{G}_{\boldsymbol{v}}(\boldsymbol{j} \boldsymbol{\omega})$ on operational frequency $\boldsymbol{f}$ in bifurcation-free region. Top - Transfer function magnitude. Bottom - Transfer function phase.

Figure 4: Voltage transfer function in bifurcation region.
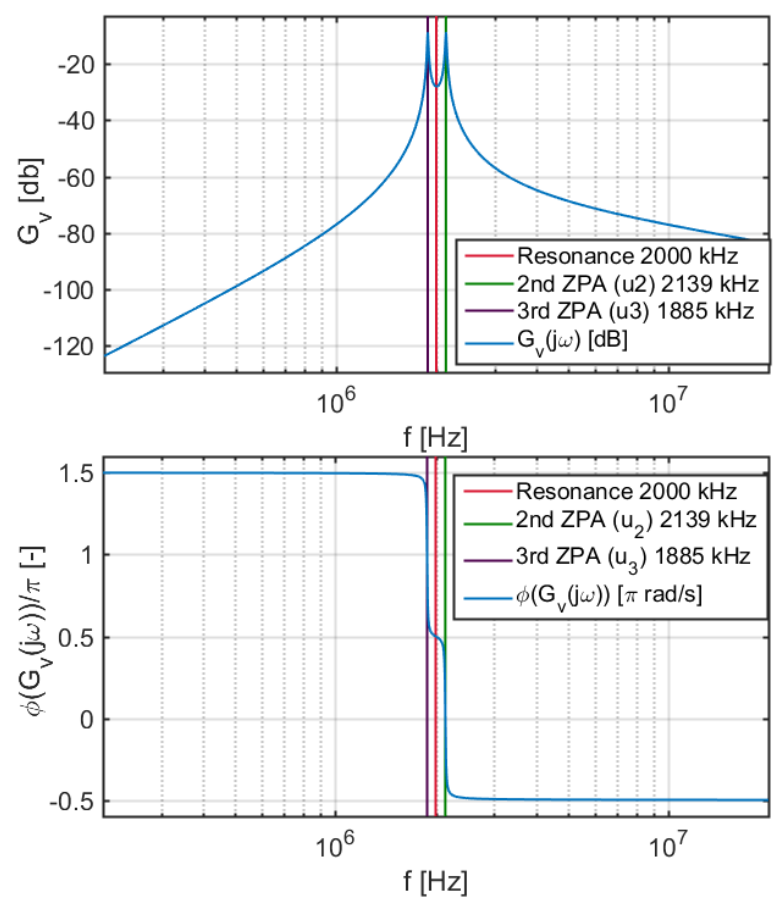

Figure shows the dependency of voltage transfer function $\boldsymbol{G}_{\boldsymbol{v}}(\boldsymbol{j} \boldsymbol{\omega})$ on operational frequency $\boldsymbol{f}$ in bifurcation-free region is depicted. Top - Transfer function magnitude. Bottom - Transfer function phase.

equal. This common resonance frequency is labeled as $f_{0}$. This condition can be also described by equation (13):

$$
\operatorname{Im}\{\hat{Z}\}=0
$$

Equation (13) allows examination of the bifurcation phenomena. The approach described in [8] is used for examination. The imaginary part of impedance is normalized by division by the real part of reflected impedance (14). Subsequently, primary winding quality factor $Q_{1}(15)$, secondary winding quality factor $Q_{2}$ (16) and normalized angular frequency $u$ (17) are substituted. Then equation (18) is obtained [8].

$$
\begin{gathered}
\operatorname{Re}\left\{Z_{r 0}\right\}=\frac{\omega_{0}^{2} M^{2}}{R_{S}} \\
Q_{1}=\frac{R_{S} L_{1}}{\omega_{0} M^{2}} \\
Q_{2}=\frac{\omega_{0} L_{2}}{R_{S}} \\
u=\frac{\omega}{\omega_{0}}=\frac{f}{f_{0}} \\
\frac{\left(u^{2}-1\right)\left[\left(Q_{1} Q_{2}^{2}-Q_{2}\right) u^{4}+\left(Q_{1}-2 Q_{1} Q_{2}^{2}\right) u^{2}+Q_{1} Q_{2}^{2}\right]}{u\left(u^{2}-1\right)^{2} Q_{2}^{2}+u^{3}}=0
\end{gathered}
$$

In our analysis, we further substitute coupling coefficient $k$ (19) from (18) and obtain equation (20).

$$
\begin{gathered}
k=\frac{M}{\sqrt{L_{1} L_{2}}}=\frac{1}{\sqrt{Q_{1} Q_{2}}} \\
\frac{\left(u^{2}-1\right)\left[\left(\frac{Q_{2}}{k^{2}}-Q_{2}\right) u^{4}+\left(\frac{1}{Q_{2} k^{2}}-2 \frac{Q_{2}}{k^{2}}\right) u^{2}+\frac{Q_{2}}{k^{2}}\right]}{u\left(u^{2}-1\right)^{2} Q_{2}^{2}+u^{3}}=0
\end{gathered}
$$

Equation (20) has six possible roots. Negative roots can be excluded as $u$ cannot be smaller than zero. Roots $u_{1}, u_{2}, u_{3}$ describe normalized frequencies (21)-(23), when impedance phase angle is equal to zero (ZPA zero phase angle). Root $u_{1}$ corresponds with resonance frequency and it is equal to 1 for whole range of normalized frequency $u$.

$$
\begin{gathered}
u_{1}=1 \\
u_{2}=\sqrt{\frac{2 \frac{Q_{2}}{k^{2}}+\sqrt{4 \frac{Q_{2}^{2}}{k^{2}}-4 \frac{1}{k^{4}}+\frac{1}{Q_{2}^{2} k^{4}}}-\frac{1}{Q_{2} k^{2}}}{2 Q_{2}\left(\frac{1}{k^{2}}-1\right)}} \\
u_{3}=\sqrt{\frac{2 \frac{Q_{2}}{k^{2}}-\sqrt{4 \frac{Q_{2}^{2}}{k^{2}}-4 \frac{1}{k^{4}}+\frac{1}{Q_{2}^{2} k^{4}}-\frac{1}{Q_{2} k^{2}}}}{2 Q_{2}\left(\frac{1}{k^{2}}-1\right)}}
\end{gathered}
$$

Roots $u_{2}, u_{3}$ are complex until $\omega_{0}$ reaches bifurcation resonance frequency $\omega_{0 b}$ and above it they are real. Frequency $\omega_{0 b}$ creates a border between bifurcation-free region $\left(\omega_{0}<\omega_{0 b}\right)$ and bifurcation region $\left(\omega_{0}>\omega_{0 b}\right)$. Its calculation (25) can be derived from condition for bifurcation-free operation for quality factors (24) [8].

$$
\begin{gathered}
Q_{1}>\frac{4 Q_{2}^{3}}{4 Q_{2}^{2}-1} \\
\omega_{0 b}=\frac{R_{S}}{L_{1} M} \sqrt{\frac{-L_{1} L_{2}^{3}\left(M^{2}-L_{1} L_{2}\right)+L_{1} L_{2}^{2}}{2}}
\end{gathered}
$$

Results of this analysis are summarized in Fig. 6. Red line illustrates bordering resonance frequency $f_{0 b}$ at which bifurcation occurs. For simulated system it is equal to $214.32 \mathrm{kHz}$. At lower frequencies the system has only one ZPA frequency, which is equal to resonance frequency (root $u_{1}$ ). However, at resonance frequencies above this border, single ZPA frequency splits into three (roots $u_{2}, u_{3}$ become real). 
Figure 6: Bifurcation map - dependency of normalized ZPA frequencies on common resonance frequency $\boldsymbol{f}_{\mathbf{0}}$.

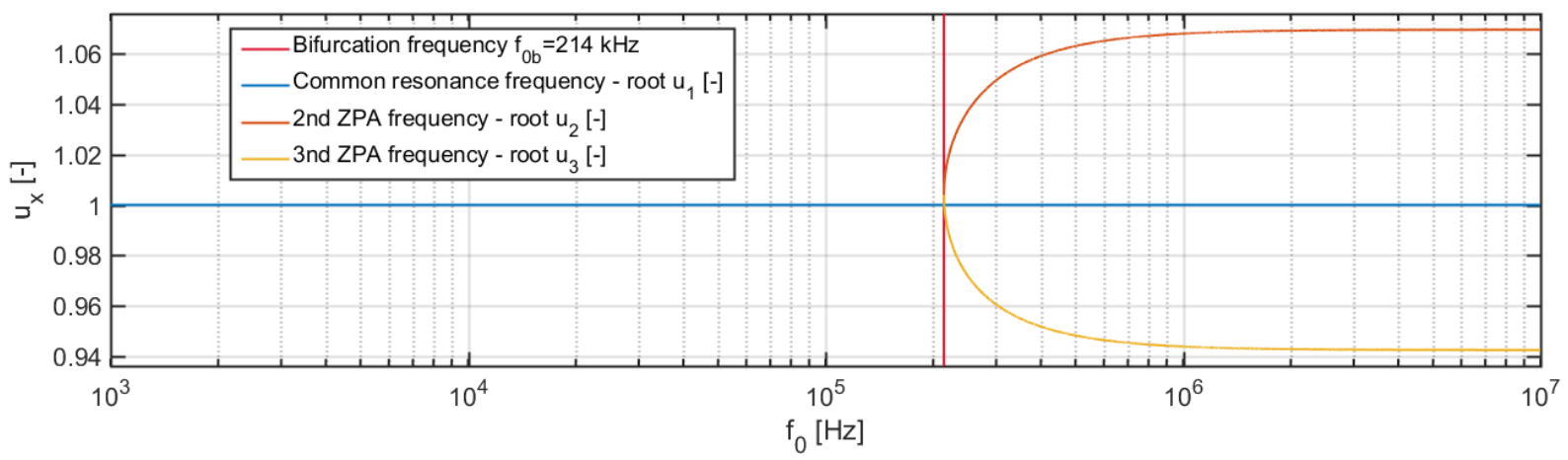

Asymptotes of $2^{\text {nd }}$ and $3^{\text {rd }}$ ZPA frequencies can be calculated as limits of roots $u_{2}$ and $u_{3}$ in infinity. They are solely dependent on coupling coefficient $k$ :

$$
\begin{aligned}
& u_{2 a}=\frac{1}{\sqrt{1-k}} \\
& u_{3 a}=\frac{1}{\sqrt{1+k}}
\end{aligned}
$$

To evaluate two-coil IPT system behavior from the perspective of bifurcation, we used impedance and voltage transfer function. Fig. 7 and Fig. 8 depict waveforms of impedance and voltage transfer function at the ZPA frequencies in dependency on the common resonance frequency $f_{0}$ (i.e. waveforms of impedance and voltage transfer function in the points corresponding to bifurcation map).

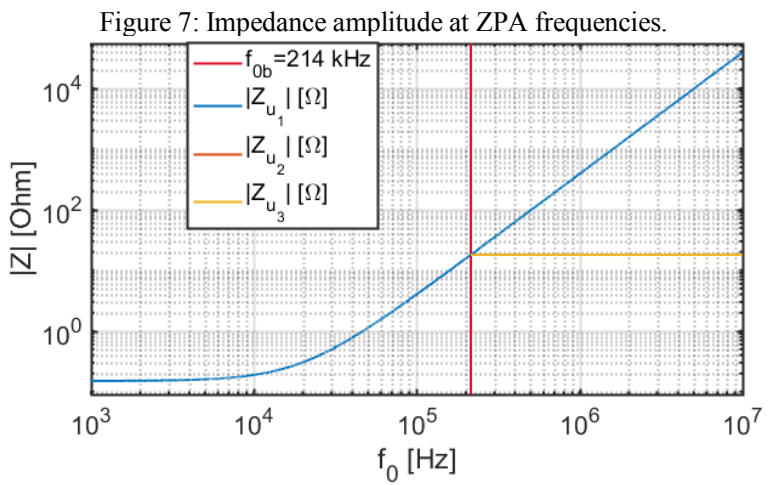

Impedance amplitude at ZPA frequencies in dependence on the common resonance frequency $\boldsymbol{f}_{\mathbf{0}}$.

Impedance amplitude at ZPA frequencies $\left|Z_{u_{x}}\right|$ in dependency on $f_{0}$ is depicted in Fig 7. In bifurcationfree region there is only one ZPA frequency $u_{1}$, which is identical to $f_{0}$. Impedance amplitude $\left|Z_{u_{1}}\right|$ at $f_{0}$ is almost constant in the beginning, then starts growing exponentially until $f_{0}$ reaches bifurcation frequency $f_{0 b}$. Impedance amplitude is equal to $18 \Omega$ at $f_{0 b}$. At this moment, bifurcation occurs and system operating point moves into bifurcation region.

Amplitude $\left|Z_{u_{1}}\right|$ continues its exponential growth. However, amplitudes $\left|Z_{u_{2}}\right|,\left|Z_{u_{3}}\right|$ at $2^{\text {nd }}$ and $3^{\text {rd }} Z \mathrm{PA}$ frequencies split from $\left|Z_{u_{1}}\right|$ and remains constant with value equal to impedance amplitude at $f_{0 b}$ (in Fig. 7 , $\left|Z_{u_{2}}\right|$ and $\left|Z_{u_{3}}\right|$ are overlapping).

Only ZPA frequencies are evaluated, therefore impedance phase remains equal to zero in all cases.
Transfer function magnitudes $G_{u_{x}}[d B]$ and phases $\varphi\left(G_{u_{x}}\right)$ are illustrated in Fig. 8. In bifurcation-free region, there is also only one ZPA frequency identical with common resonance frequency. At first, magnitude $G_{u_{1}}[d B]$ is exponentially growing, until it reaches a plateau with peak $6.11 \mathrm{~dB}$ at $19.69 \mathrm{kHz}$. With further increase of $f_{0}$, the magnitude starts exponentially decreasing, until $f_{0}$ reaches bifurcation at $f_{0 b}$. Magnitude is equal to $-8.70 \mathrm{~dB}$ at $f_{0 b}$. Transfer function phase for $u_{1}$ is equal to $\frac{\pi}{2}$ in bifurcation-free region.

Figure 8: Transfer function at ZPA frequencies.
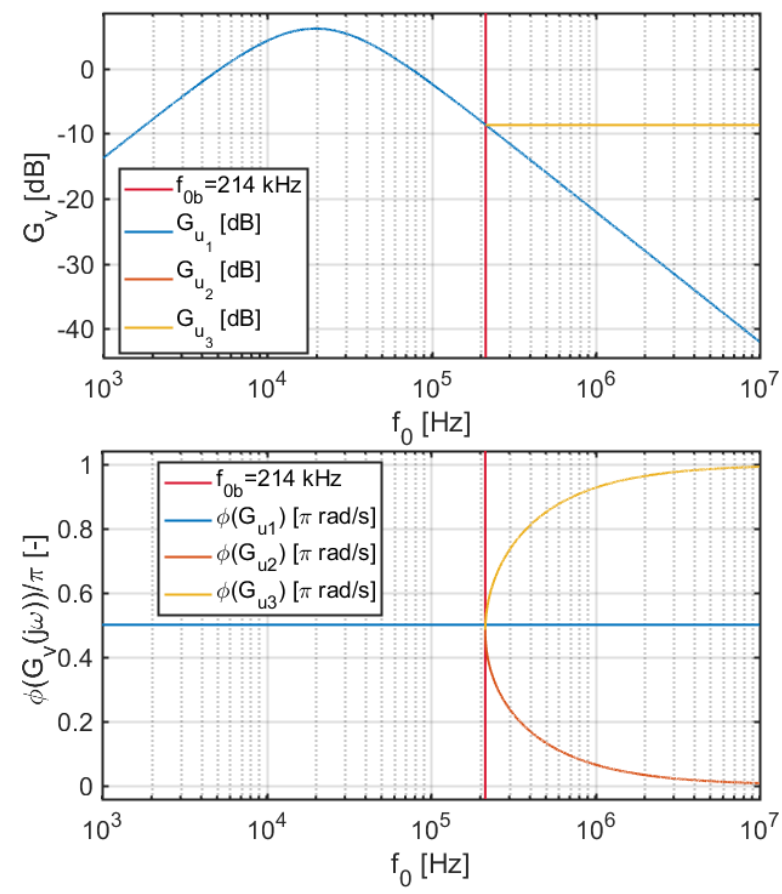

Transfer function at ZPA frequencies in dependence on the common resonance frequency $\boldsymbol{f}_{\mathbf{0}}$. Top - Transfer function magnitude. Bottom - Transfer function phase.

When system operating point moves to bifurcation region, ZPA frequencies $u_{2}, u_{3}$ split off the $u_{1}$ which corresponds with common resonance frequency. Further increase of resonant frequency in bifurcation region doesn't affect transfer function magnitudes $G_{u_{2}}[d B], G_{u_{3}}[d B]$, it only allows stabilization of phases for roots $u_{2}, u_{3}$ at 0 or $\pi$ respectively. However, magnitude $G_{u_{1}}[d B]$ for $u_{1}$ keeps its exponential decline with the same slope as it has at $f_{0 b}$. Phase $\varphi\left(G_{u_{1}}\right)$ for $u_{1}$ remains equal to $\frac{\pi}{2}$, as in bifurcation-free region. 
This analysis shows that in bifurcation-free region the IPT system shall be operated at frequency given by root $u_{1}$ (equal to common resonance frequency). In bifurcation-free region, the amplitude of output voltage $v_{o}$ can be controlled by choice of resonance frequency (Fig. 8 Top). However, in bifurcation region, it is convenient to abandon common resonance frequency $f_{0}$ and choose frequency given by root $u_{2}$ or root $u_{3}$, as transfer function magnitude for $u_{1}$ keeps dropping. The amplitude of output voltage $v_{o}$ remains constant in whole bifurcation area for $u_{2}, u_{3}$. If maximal amplification of the output voltage is desired, this particular system should be operated in bifurcation-free area at approximate frequency of $19.69 \mathrm{kHz}$.

\section{CONCLUSION}

In this paper, bifurcation in dependency on resonance frequency was examined. At first, two-coil IPT system was described, and its voltage equations derived. Then impedance and transfer function equations were obtained. This laid a foundation for bifurcation analysis. The main result of this analysis is a bifurcation map which visualizes bifurcation phenomena in dependency on frequency, and equations, which allows calculation of this map from IPT system parameters

This analysis also shows the importance of resonance frequency choice for output voltage amplification.

\section{FUTURE WORK}

Attributes, such as bifurcation resonance frequency $f_{0 b}$, values of impedance amplitude and transfer function magnitude for $u_{2}, u_{3}$ in bifurcation region and transfer function magnitude peak in bifurcation-free region are dependent on IPT system parameters, such as $k, Q_{1}, Q_{2}$ and therefore they can be controlled by them. Further analysis of these dependencies could improve design the of IPT system for desired type of operation.

\section{ACKNOWLEDGMENT}

The research described in this paper was supported by the Grant Agency of the Czech Technical University in Prague under grants No. SGS15/197/OHK3/3T/13 and No. SGS17/088/OHK3/1T/13.

\section{REFERENCES}

[1] M. P. Kazmierkowski and A. J. Moradewicz, "Unplugged But Connected: Review of Contactless Energy Transfer Systems," IEEE Industrial Electronics Magazine, vol. 6, no. 4, pp. 47-55, Dec. 2012.

[2] X. Lu, P. Wang, D. Niyato, D. I. Kim, and Z. Han, "Wireless Charging Technologies: Fundamentals, Standards, and Network Applications," IEEE Communications Surveys Tutorials, vol. 18, no. 2, pp. 1413-1452, 2016.

[3] M. G. Roes, J. L. Duarte, M. A. Hendrix, E. Lomonova, and others, "Acoustic energy transfer: a review," Industrial Electronics, IEEE Transactions on, vol. 60, no. 1, pp. 242-248, 2013.

[4] K. Takayama, S. Hiramatsu, and M. Shiho, "CW $100 \mathrm{MW}$ microwave power transfer in space," in Particle Accelerator Conference, 1991. Accelerator Science and Technology., Conference Record of the 1991 IEEE, 1991, pp. 2625-2627.

[5] G. A. Covic and J. T. Boys, "Inductive Power Transfer," Proceedings of the IEEE, vol. 101, no. 6, pp. 1276-1289, Jun. 2013.

[6] G. A. Covic and J. T. Boys, "Modern Trends in Inductive Power Transfer for Transportation Applications," IEEE Journal of Emerging and Selected Topics in Power Electronics, vol. 1, no. 1, pp. 28-41, Mar. 2013.

[7] N. Tesla, "System of transmission of electric energy," U.S. Patent 645 57620-Mar-1900.

[8] C.-S. Wang, G. A. Covic, and O. H. Stielau, "Power transfer capability and bifurcation phenomena of loosely coupled inductive power transfer systems," Industrial Electronics, IEEE Transactions on, vol. 51, no. 1, pp. 148-157, Feb. 2004.

[9] A. Kurs, A. Karalis, R. Moffatt, J. D. Joannopoulos, P. Fisher, and M. Soljačić, "Wireless power transfer via strongly coupled magnetic resonances," science, vol. 317, no. 5834, pp. 83-86, 2007.

[10] A. P. Sample, D. T. Meyer, and J. R. Smith, "Analysis, Experimental Results, and Range Adaptation of Magnetically Coupled Resonators for Wireless Power Transfer," IEEE Transactions on Industrial Electronics, vol. 58, no. 2, pp. 544554, Feb. 2011. 